\title{
Multiple subtentorial metastasis in diffuse midline glioma receiving tumor treating fields: a case report and literature review
}

\author{
Chaofeng Liang ${ }^{1 \#}$, Jin Gong ${ }^{1 \#}$, Baoyu Zhang ${ }^{1}$, Zhan'ao Meng $^{2}$, Manting Li $^{1}$, Ying Guo ${ }^{1 \#}$ \\ ${ }^{1}$ Department of Neurosurgery, 3rd Affiliated Hospital of Sun Yat-sen University, Sun Yat-sen University, Guangzhou, China; ${ }^{2}$ Department of \\ Radiology, 3rd Affiliated Hospital of Sun Yat-sen University, Sun Yat-sen University, Guangzhou, China \\ \#These authors contributed equally to this work. \\ Correspondence to: Chaofeng Liang; Ying Guo. Department of Neurosurgery, 3rd Affiliated Hospital of Sun Yat-sen University, Sun Yat-sen \\ University, Guangzhou 510630, China. Email: 1cfjeff@163.com; guoy@mail.sysu.edu.cn.
}

\begin{abstract}
Diffuse midline glioma (DMG) is one of fatal glioblastoma multiforme (GBM) with no proven medical therapies. Tumor treating fields (TTFields) is a new revolutionary therapy for GBM which prolongs the overall survival time obviously. However, we can observe more tumor growth phenomena (such as distant multiple metastases) than before. This report describes an adult patient who presented headache and dizziness, accompanied by left limb weakness, nausea, and vomiting following car accident trauma, following imaging examinations suggested thalamus GBM. He was treated with subtotal excision. Final pathology was diagnosed as DMG with H3F3A mutation, isocitrate dehydrogenase (IDH) wild type. Following concurrent chemoradiation therapy (CCRT) and adjuvant temozolomide (TMZ) chemotherapy + TTFields therapy were carried out. Supratentorial tumor has been exhibited a partial radiological response for nine months until TTFields was used irregularly or even discontinued in the later stage. Especially, subtentorial and spinal multiple metastasis occurred during this time. Both supratentorial and subtentorial tumors were treated with surgery, radiotherapy, chemotherapy, even targeted drugs, with the only difference being TTFields, but we could see different consequences for tumor growth. One conclusion might be drawn that TTFields can provide a longer survival time (14 vs. 8 months reported before) for DMG patients and improve survival benefits. However, we can observe that patients maybe die from subtentorial metastasis because TTFields could not cover the subtentorial tumors, which is the focal challenge at present. So further research on subtentorial tumors with TTFields is urgently needed.
\end{abstract}

Keywords: Diffuse midline glioma (DMG); tumor treating fields (TTFields); subtentorial metastasis; case report

Submitted Jul 30, 2021. Accepted for publication Oct 16, 2021.

doi: 10.21037/atm-21-4395

View this article at: https://dx.doi.org/10.21037/atm-21-4395

\section{Introduction}

Diffuse midline glioma (DMG) is one of fatal glioblastoma multiforme (GBM) with no standard effective medical therapies. GBM treatment consists of surgery, radiotherapy, and chemotherapy with temozolomide (TMZ) (1). In 2018, the new version of National Comprehensive Cancer Network (NCCN) guidelines clearly indicated tumor treating fields (TTFields) therapy as a category 1 recommendation (2). As a result, patients can live longer after surgery with 'concurrent chemoradiation therapy (CCRT) and adjuvant TMZ chemotherapy + TTFields therapy'.

Patients can benefit from longer overall survival (OS) with TTFields, which increased from 16 to 20.9 months (3), but some new and rare challenges have emerged, among

\footnotetext{
$\wedge$ ORCID: 0000-0001-5975-3320.
} 
which distant metastasis to the central nervous system (CNS) has been reported only occasionally in the past. This case describes a patient who exhibited a partial radiological response of the supratentorial tumor following initiation of TTFields therapy for 9 months, but showed subtentorial and spinal multiple metastasis 4 months after beginning TTFields treatment. TTFields can provide a longer survival time (14 months) in this case than previous report (8 months) for DMG patients (4). However, patients maybe die from subtentorial metastasis at last because the coverage area of TTFields, which is generally effective for supratentorial tumors, but may not applying to subtentorial tumors.

We present the following article in accordance with the CARE reporting checklist (available at https://dx.doi. org/10.21037/atm-21-4395).

\section{Case presentation}

All procedures performed in studies involving human participants were in accordance with the ethical standards of the institutional and/or national research committee(s) and with the Helsinki Declaration (as revised in 2013). Written informed consent was obtained from the patient for publication of this case report and accompanying images. A copy of the written consent is available for review by the editorial office of this journal.

A 28-year-old male patient presented headache and dizziness, accompanied by left limb weakness, nausea, and vomiting following car accident trauma. On the initial physical examination, the left limb strength was grade 4 and hypoesthesia. Without abnormal family and medical history. Subsequently, he underwent imaging examinations, and both skull computerized tomography (CT) and magnetic resonance imaging (MRI) suggested a mass with hemorrhage in the right thalamus (Figure 1). The mass was heterogeneous with different composition, as well as irregular necrotic cystic areas with enhancement. The preoperative three-dimensional reconstruction of lesions and surrounding white matter fiber bundles showed that the main functional fiber bundles were pushed to the peripheral and anterior upper parts of the lesions.

Next, the patient underwent a right thalamic tumor resection assisted by ultrasonic navigation and electrophysiological monitoring, and the necrotic and cystic areas of the tumor were removed. The ventricle was opened intraoperatively and an external drainage tube was placed for drainage. The patient was pathologically diagnosed with GBM (Figure 2). Tumor gene analysis showed non-methylation of the O6-methylguanine-DNA methyltransferase (MGMT) promoter, no $1 \mathrm{p} / 19 \mathrm{q}$ codeletion, isocitrate dehydrogenase (IDH) wild type, and human telomerase reverse transcriptase [TERT(-)].

The patient was initially treated with CCRT, which involved initial loading with TMZ concurrently with conformal radiation therapy (60 Gy) followed by monthly TMZ. TTFields was initiated after two cycles of TMZ adjuvant therapy, and the TTFields utilization rate was $83 \%$ until 8 months postoperatively. The patient exhibited a partial radiological response according to the 2010 response assessment in neuro-oncology (RANO) standard (Figure 3). The patient developed symptoms of lumbago, leg pain, and defecation weakness 9 months after the initial operation. MRI revealed metastatic tumors on the L5 spinal cord. The tumor was found to be surrounding the cauda equina nerve and was completely removed (Figure 4).

Postoperative pathological examination reported GBM metastasis, and the tumor gene analysis results were consistent with those of the thalamic tumor (Figure 5). In addition, H3F3A mutation was found, which was diagnosed as DMG. H3F3A-mutant DMG is a fatal malignancy with no proven medical therapies. ONC201 is a small molecule selective antagonist of dopamine receptor D2/3 (DRD2/3) with an exceptional safety profile. Following up on a durable response in the first $\mathrm{H} 3 \mathrm{~F} 3 \mathrm{~A}-$ mutant DMG patient who received ONC201 (NCT02525692), an expanded clinical study suggests that 3 adults among the 14 recurrent patients remain on treatment progression-free with a median follow up of 49.6 weeks. Radiographic regressions were reported in a subset of patients with thalamic and pontine gliomas, along with improvements in disease-associated neurological symptoms (5). In addition, CDK6 gene amplification was detected, and it has been reported that GBM cell lines carrying CDK6 gene amplification were sensitive to palbociclib (6). While palbociclib was only approved by FDA as a potential benefit drug for other cancers.

Subsequently, the patient received local radiation therapy $(54 \mathrm{~Gy} / 27 \mathrm{~F})$ in the lumbar tumor region. However, 1 month after the operation, four new metastases were found in the cervical 6 and lumbar 2-4 spinal cord (Figure 6), so the cervical spinal metastasis was treated with palliative radiotherapy (46 Gy/23 F). Meanwhile, the patient also received systemic chemotherapy: bevacizumab $600 \mathrm{mg}$ (D1) + palbociclib $125 \mathrm{mg} /$ day (D1-21). However, palbociclib was discontinued because the patients could not tolerate the side effects, especially myelosuppression 

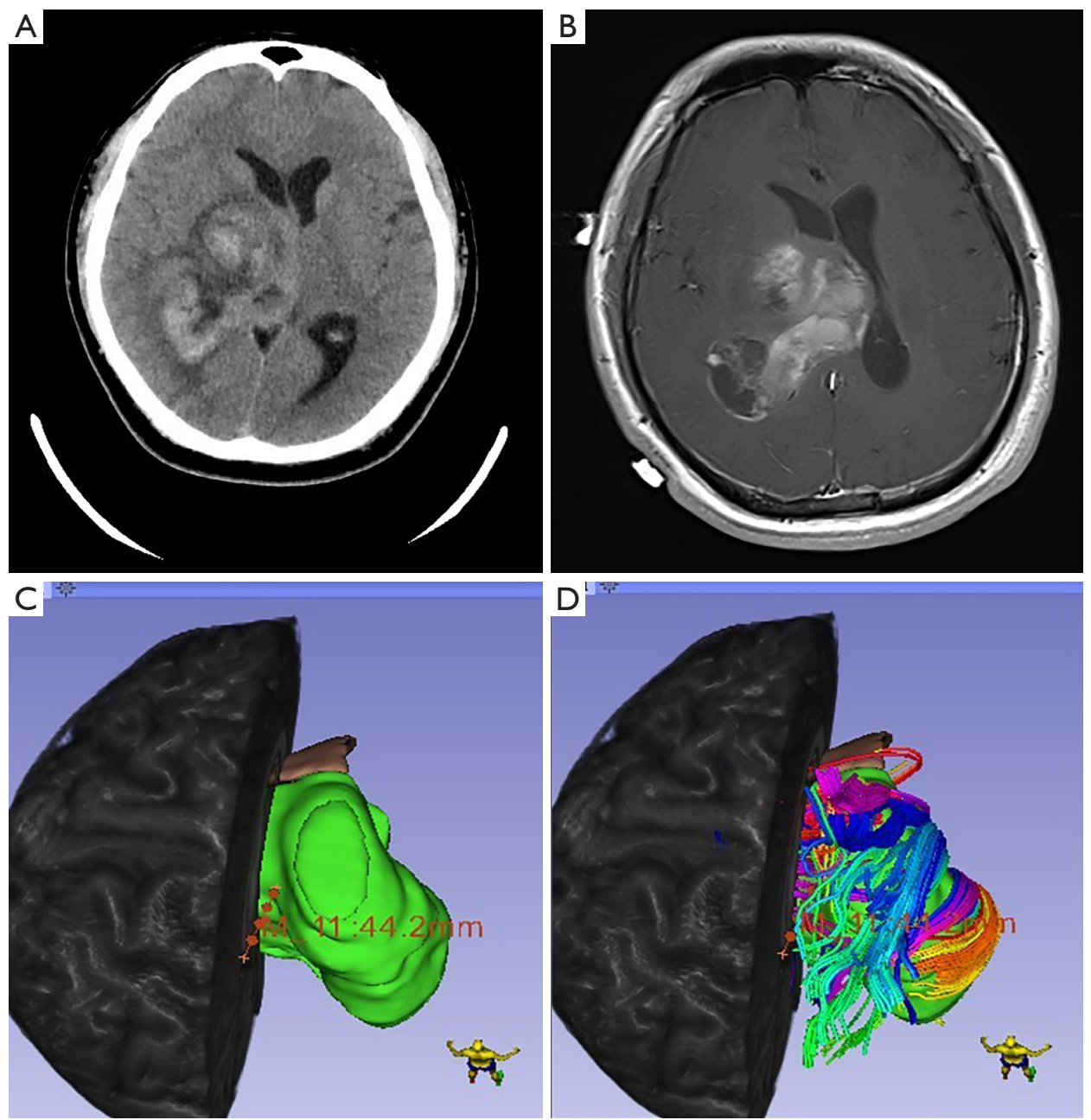

Figure 1 Preoperative imaging and 3D reconstruction. (A) Preoperative CT showing tumor and hemorrhage on the right side of the thalamus. (B) Preoperative MRI T1 with gadolinium contrast showed a typical contrast enhancement pattern of GBM-associated hemorrhage and necrosis. (C,D) 3D Slicer software was used to reconstruct the relationship between the tumor and the ventricle and surrounding white matter tracts, which indicated that important functional fiber bundles encircled the tumor. CT, computed tomography; MRI, magnetic resonance imaging; GBM, glioblastoma multiforme.

and gastrointestinal reactions.

Due to COVID-19, the supply of TTFields accessories has been interrupted, resulting in patients having to discontinuously use TTFields from lumbar metastasis surgery to 1 week prior to radiotherapy, during that time the TTFields utilization rate decreased to $78 \%$. The following month, TTFields had to be terminate due to lack of accessories. MRI showed that the tumor in the right thalamus had developed, and a new metastatic tumor appeared in cerebellopontine angle (Figure 7). Two months later, the patient died.

\section{Discussion}

The median OS of different genotypes of GBM varies, which has been reported in literature to be between 13.5 and 16.6 months (7). Our patient was classified as IDH wild type, no $1 \mathrm{P} / 19 \mathrm{Q}$ co-deletion, and without TERT mutations; the median OS for this type should be $>16.6$ months. Judging by the imaging changes of the primary thalamic tumor in this patient, the cause of death was not the recurrence and enlargement of the primary thalamic tumor, but the metastatic spread throughout the CNS, especially the brainstem and cervical spinal cord metastases. These may 
Page 4 of 9

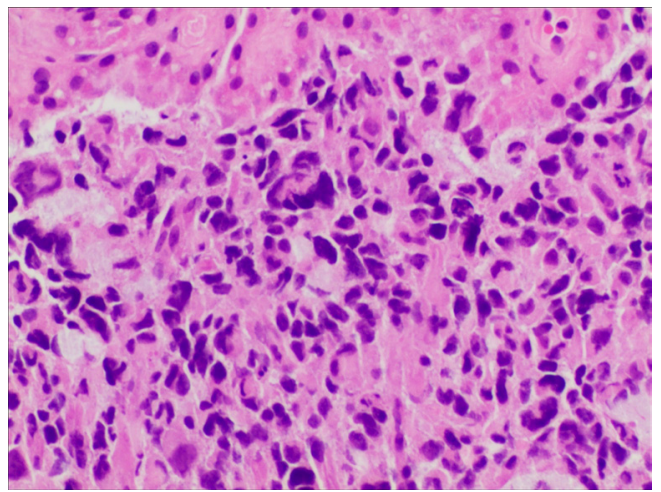

Figure 2 Light micrographs of the glioma. Tissue stained with hematoxylin and eosin ( $\mathrm{H} \& \mathrm{E})$ showing diffuse and densely distributed tumor cells. Nuclear atypia was obvious (bar $=50 \mu \mathrm{m})$. have affected the patient's breathing and lead to death due to dyspnea (8).

Despite the combination of potentially effective targeted drug therapies after the second surgical resection of the intraspinal metastases, the patient was not able to tolerate the side effects of multiple drugs, resulting in poor evaluation of the efficacy of these drugs. For this patient's metastatic tumor of entire CNS, we considered there may be two main reasons. (I) H3F3A mutation. H3F3A mutation is generally considered to be a character of DMG, which is mainly grows in the midline, and an important factor with poor prognosis $(9,10)$. Patients diagnosed as DMG with $\mathrm{H} 3 \mathrm{~F} 3 \mathrm{~A}$ mutation was reported OS was approximately 9.4 months (11). Even with individualized targeted drug
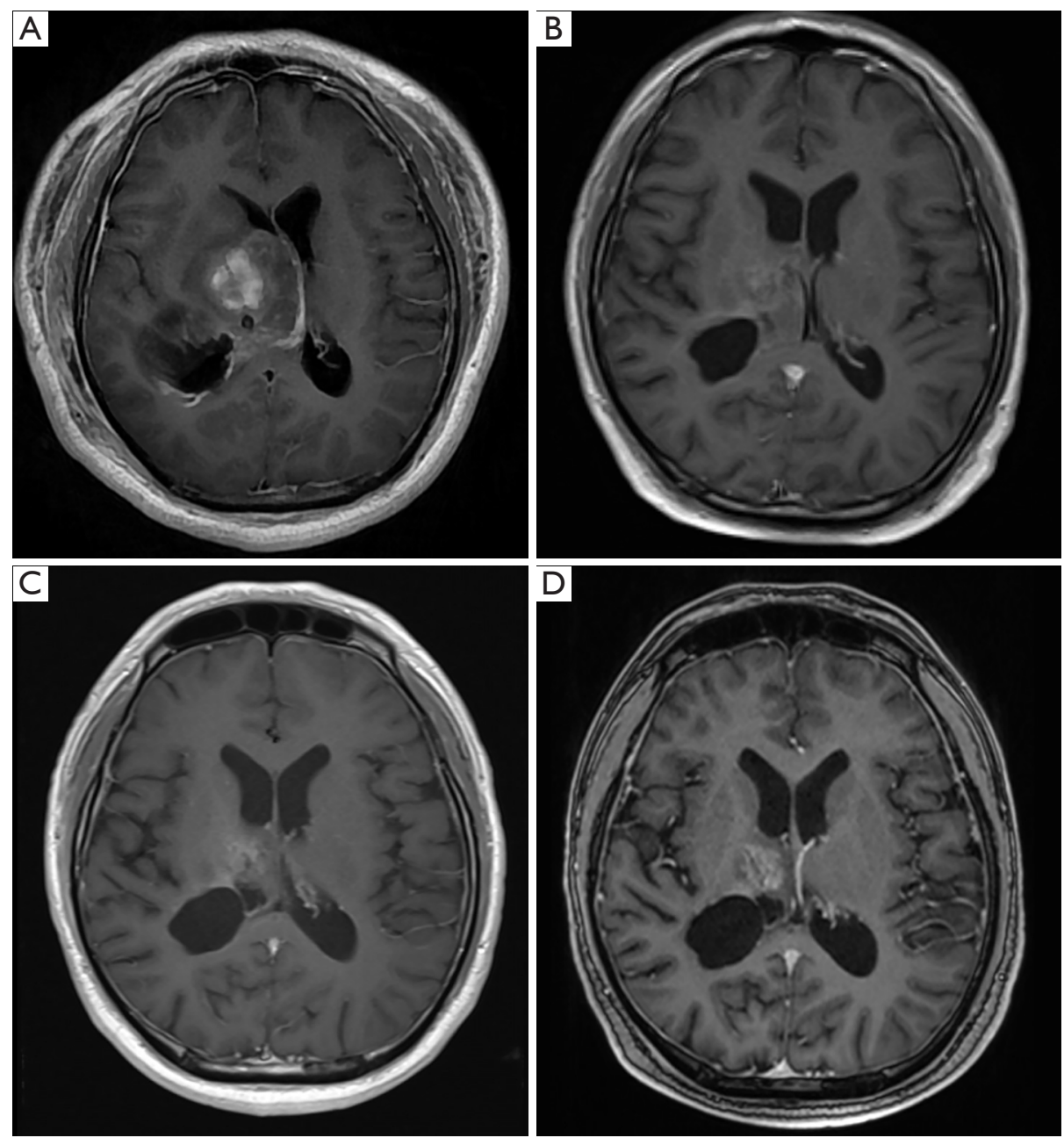

Figure 3 Postoperative MRI. (A) 72 h; (B) 2 months; (C) 5 months; (D) 8 months after surgery showing that the tumor was stable on enhanced MRI T1 sequence. MRI, magnetic resonance imaging. 

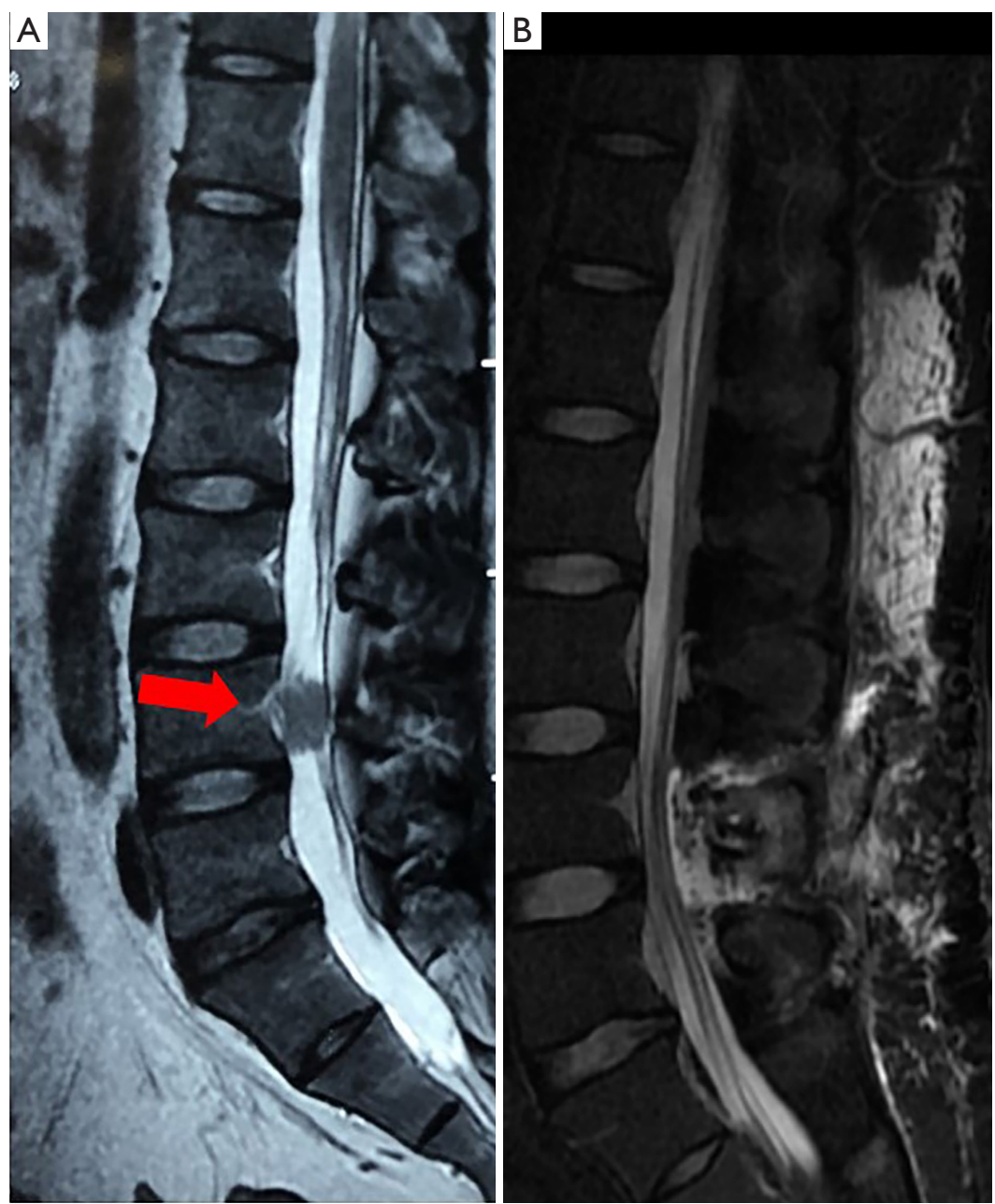

Figure 4 Pre- and post-operative MRI in second surgery. (A) MRI T2 sequence showing a metastatic tumor (red arrow) on the L5 spinal cord pressing on the surrounding nerves 9 months after the initial operation; (B) postoperative T2 sequences showing that the tumor was completely removed. MRI, magnetic resonance imaging.

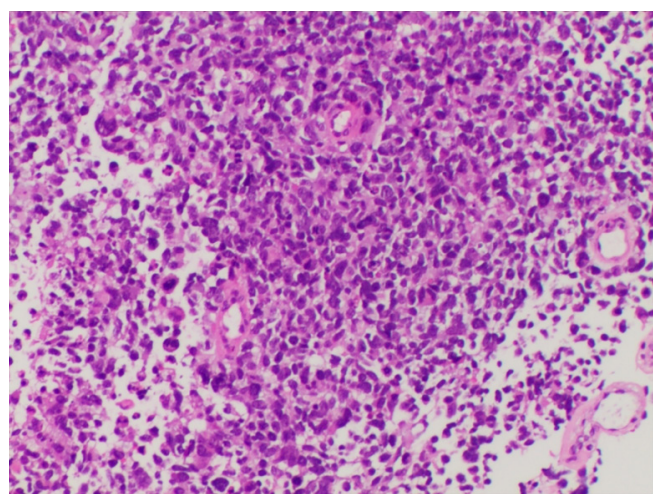

Figure 5 Light micrographs of the glioma. Tissue stained with $\mathrm{H} \& \mathrm{E}$ showing microvascular proliferation and necrosis (bar $=200 \mu \mathrm{m}$ ). therapy, the prognosis is still poor. Gojo reported 12 cases of DMG patients who received personalized treatment, including inhibition of the PI3K/AKT/mTOR pathway, mitogen-activated protein kinase (MAPK) signaling, immunotherapy, receptor tyrosine kinase inhibition, and retinoic receptor agonist. The median time to progression in these patients was 29 weeks, and the median OS was 16.5 months (12). (II) Ventricle opening. In theory, the tumor cells will circulate along the CSF, carrying the tumor to other CNS sites, however, there is few case reported glioma with CNS metastasis before TTFields application. Of course, we can't rule out that the OS is prolonged after TTFields application, so we can observe more complication 

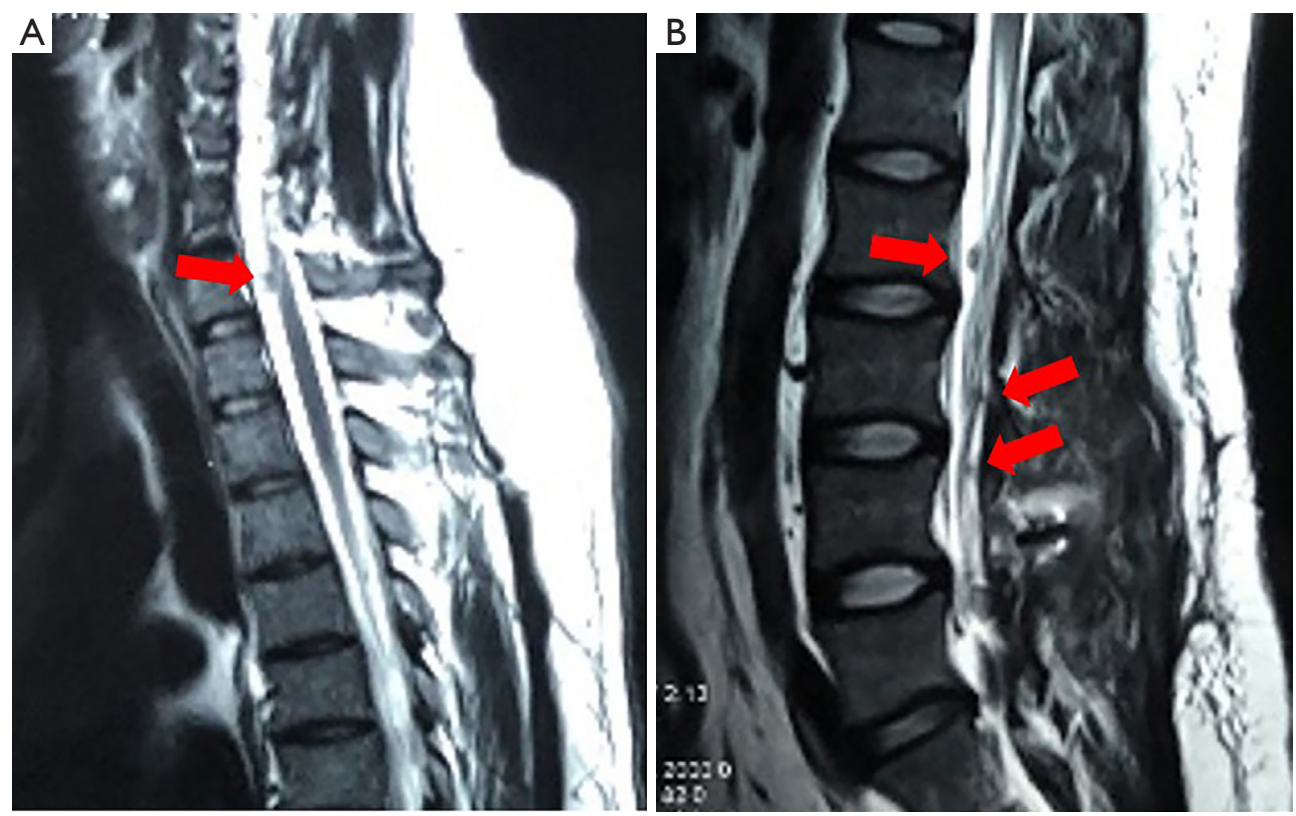

Figure 6 MRI T2 sequences showing four new metastases (red arrow) in the cervical 6 and lumbar 2-4 spinal cord. MRI, magnetic resonance imaging.
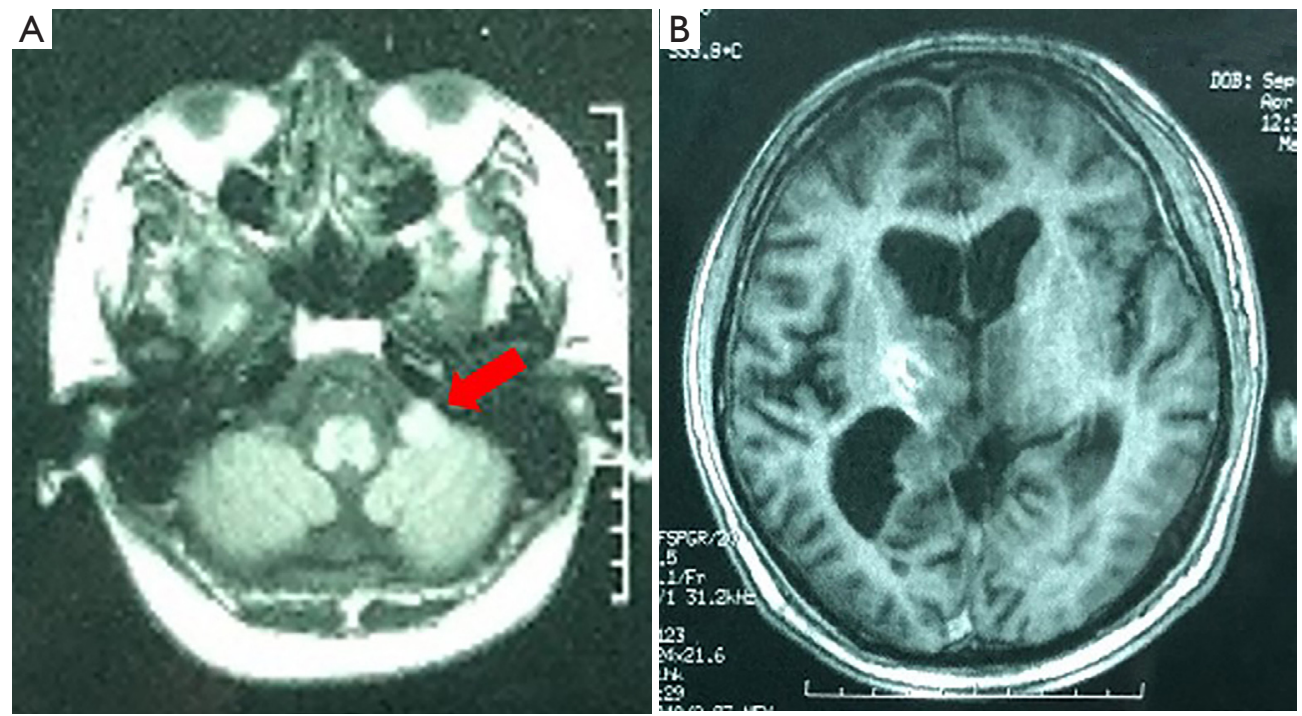

Figure 7 MRI T1 with contrast showing a new metastasis (red arrow) in the cerebellopontine angle and the enlarged original tumor in the right thalamus. MRI, magnetic resonance imaging.

that were not observed before.

With regards to the patient's entire treatment process, TTFields was very effective for primary thalamic tumor (13). This may be attributable to the fact that the thalamic tumor was stable when the patient was treated with TTFields continuously in the early stage, and recurred when the
TTFields was used irregularly or even discontinued in the later stage. In the EF-14 study, the Asian subgroup also showed efficacy and safety consistent with the general population. Kim reported that newly diagnosed GBM (ndGBM) patients in Korea participating in the EF-14 trial were similar to the entire EF-14 population (14). 
However, TTFields could not cover the lesions under the tentorium, which is the focal challenge at present. TTFields has been approved by the FDA for the treatment of relapsed or ndGBM in the key clinical studies EF-11 and EF-14, both of which are supratentorial tumors (15). Therefore, the indications are only for supratentorial GBM. There are no large clinical trials of TTFields in supratentorial tumors, and only exploratory studies are available for reference. Professor Edwin investigated a series of alternative array configurations for the posterior fossa to determine the electric field coverage of a cerebellar GBM. The posteroanterior (PA) horizontal arrangement provided an average $46.6 \%$ increase in gross tumor volume (GTV) compared to supratentorial array when the PA array was placed initiating along the patient's posterior neck and horizontally parallel, along the longer axis, to the coronal plane of the patient's head (16). Further clinical studies of TTFields for subtentorial tumors are expected in the future.

In this case, the tumor in the primary thalamic site was stabled when the utilization rate of TTFields was high in the early stage, while the tumor recurred significantly when the utilization rate decreased or even stopped. Professor Toms has suggested that efficacy of TTFields was positively correlated with duration of use (17). In the study, TTFields were most effective when used for more than 18 hours per day. The median OS of patients with was 25 months ( $\geq 22$ hours per day), and the 5 -year OS increased from $4.5 \%$ to $29.3 \%$. Therefore, some scholars propose to start using TTFields with CCRT as soon as possible. Currently, a small sample study shows that TTFields is safe to be used simultaneously in the stage of CCRT $(18,19)$. A large phase III RCT study of ndGBM in the stage of CCRT combined with TTFields is compared with that without TTFields (EF-32, NCT: 04471844) is also in progress. The application timing of TTFields awaits further data generation in the future.

In addition, this case shows that patients with a skin disorder may tolerate TTFields treatment well, with a good quality of life (QOL) and no severe skin reactions to the therapy. However, metastasis in other areas result in corresponding nerve root symptoms, leading to the deterioration of the QOL of patients (20). In the EF-14 study of ndGBM, TTFields in combination with TMZ was found to significantly improve survival versus TMZ alone (17). To achieve an optimal response, TTFields therapy should be used on a continuous basis for a minimum of 18 hours a day. Although patients almost experienced TTFields-related skin adverse reactions. In a retrospective safety review of TTFields after marketing (10/2011-2/2019) (21), 63\% of 11029 patients with brain tumors treated with TTFields reported $\geq 1$ AE. Stratified by diagnosis, $65 \%$ of ndGBM, $60 \%$ of recurrent GBM (rGBM), $65 \%$ of anaplastic astrocytoma and anaplastic oligodendroglioma, and $61 \%$ of other reported brain tumors reported $\geq 1 \mathrm{AE}$. When stratified by age, $58 \%$ of children, $63 \%$ of adults, and $66 \%$ of elderly patients reported $\geq 1 \mathrm{AE}$. Overall, AEs with an incidence of $\geq 5 \%$ reported in all cohorts was comparable in diagnosis and age subgroups. Skin disorders (36\%), general/application site disorders $(31 \%)$, and neurological disorders $(27 \%)$ were the most frequently reported adverse reactions among all patients, with similar rates in diagnosis and age subgroups. There no other TTFields-related toxicities were observed without an increase in radiation therapy or TMZ related toxicities as a result of combining TTFields with these therapies.

TTFields is a promising therapeutic approach for GBM patients, but there are still difficulties for subtentorial tumor, which requires further exploration and research. However, the significance of individualized targeted therapy is not clear, and the number of cases needs to be further expanded to obtain a more precise conclusion.

\section{Acknowledgments}

Funding: The present study was supported by the Sun Yat-sen University Clinical Research 5010 Program (No. 2018025) and Science and Technology Planning Project of Guangzhou (202102010421).

\section{Footnote}

Reporting Checklist: The authors have completed the CARE reporting checklist. Available at https://dx.doi. org/10.21037/atm-21-4395

Conflicts of Interest: All authors have completed the ICMJE uniform disclosure form (available at https://dx.doi. org/10.21037/atm-21-4395). The authors have no conflicts of interest to declare.

Ethical Statement: The authors are accountable for all aspects of the work in ensuring that questions related to the accuracy or integrity of any part of the work are appropriately investigated and resolved. All procedures performed in studies involving human participants were in 
accordance with the ethical standards of the institutional and/or national research committee(s) and with the Helsinki Declaration (as revised in 2013). Written informed consent was obtained from the patient for publication of this case report and accompanying images. A copy of the written consent is available for review by the editorial office of this journal.

Open Access Statement: This is an Open Access article distributed in accordance with the Creative Commons Attribution-NonCommercial-NoDerivs 4.0 International License (CC BY-NC-ND 4.0), which permits the noncommercial replication and distribution of the article with the strict proviso that no changes or edits are made and the original work is properly cited (including links to both the formal publication through the relevant DOI and the license). See: https://creativecommons.org/licenses/by-nc-nd/4.0/.

\section{References}

1. Stupp R, Hegi ME, Mason WP, et al. Effects of radiotherapy with concomitant and adjuvant temozolomide versus radiotherapy alone on survival in glioblastoma in a randomised phase III study: 5 -year analysis of the EORTC-NCIC trial. Lancet Oncol 2009;10:459-66.

2. Rick J, Chandra A, Aghi MK. Tumor treating fields: a new approach to glioblastoma therapy. J Neurooncol 2018;137:447-53.

3. Stupp R, Taillibert S, Kanner A, et al. Effect of TumorTreating Fields Plus Maintenance Temozolomide vs Maintenance Temozolomide Alone on Survival in Patients With Glioblastoma: A Randomized Clinical Trial. JAMA 2017;318:2306-16.

4. Hassan U, Latif M, Yousaf I, et al. Morphological Spectrum and Survival Analysis of Diffuse Midline Glioma With H3K27M Mutation. Cureus 2021;13:e17267.

5. Chi AS, Tarapore RS, Hall MD, et al. Pediatric and adult H3 K27M-mutant diffuse midline glioma treated with the selective DRD2 antagonist ONC201. J Neurooncol 2019;145:97-105.

6. Michaud K, Solomon DA, Oermann E, et al. Pharmacologic inhibition of cyclin-dependent kinases 4 and 6 arrests the growth of glioblastoma multiforme intracranial xenografts. Cancer Res 2010;70:3228-38.

7. Ma S, Rudra S, Campian JL, et al. Prognostic impact of CDKN2A/B deletion, TERT mutation, and EGFR amplification on histological and molecular IDH-wildtype glioblastoma. Neurooncol Adv 2020;2:vdaa126.
8. Goryaynov SA, Potapov AA, Ignatenko MA, et al. Glioblastoma metastases: a literature review and a description of six clinical observations. Zh Vopr Neirokhir Im N N Burdenko 2015;79:33-43.

9. Karremann M, Gielen GH, Hoffmann M, et al. Diffuse high-grade gliomas with $\mathrm{H} 3 \mathrm{~K} 27 \mathrm{M}$ mutations carry a dismal prognosis independent of tumor location. Neuro Oncol 2018;20:123-31.

10. La Rocca G, Sabatino G, Altieri R, et al. Significance of H3K27M Mutation in "Nonmidline" High-Grade Gliomas of Cerebral Hemispheres. World Neurosurg 2019;131:174-6.

11. Wierzbicki K, Ravi K, Franson A, et al. Targeting and Therapeutic Monitoring of H3K27M-Mutant Glioma. Curr Oncol Rep 2020;22:19.

12. Gojo J, Pavelka Z, Zapletalova D, et al. Personalized Treatment of H3K27M-Mutant Pediatric Diffuse Gliomas Provides Improved Therapeutic Opportunities. Front Oncol 2020;9:1436.

13. Stein M, Dohmen H, Wölk B, et al. Case Report of Complete Radiological Response of a Thalamic Glioblastoma After Treatment With Proton Therapy Followed by Temozolomide and Tumor-Treating Fields. Front Oncol 2020;10:477.

14. Kim CY, Paek SH, Nam DH, et al. Tumor treating fields plus temozolomide for newly diagnosed glioblastoma: a sub-group analysis of Korean patients in the EF-14 phase 3 trial. J Neurooncol 2020;146:399-406.

15. Stupp R, Wong ET, Kanner AA, et al. NovoTTF-100A versus physician's choice chemotherapy in recurrent glioblastoma: a randomised phase III trial of a novel treatment modality. Eur J Cancer 2012;48:2192-202.

16. Lok E, San P, Liang O, et al. Finite element analysis of Tumor Treating Fields in a patient with posterior fossa glioblastoma. J Neurooncol 2020;147:125-33.

17. Toms SA, Kim CY, Nicholas G, et al. Increased compliance with tumor treating fields therapy is prognostic for improved survival in the treatment of glioblastoma: a subgroup analysis of the EF-14 phase III trial. J Neurooncol 2019;141:467-73.

18. Bokstein F, Blumenthal D, Limon D, et al. Concurrent Tumor Treating Fields (TTFields) and Radiation Therapy for Newly Diagnosed Glioblastoma: A Prospective Safety and Feasibility Study. Front Oncol 2020;10:411.

19. Song A, Bar-Ad V, Martinez N, et al. Initial experience with scalp sparing radiation with concurrent temozolomide and tumor treatment fields (SPARE) for patients with newly diagnosed glioblastoma. J Neurooncol 
2020;147:653-61.

20. Onken J, Goerling U, Heinrich M, et al. Patient Reported Outcome (PRO) Among High-Grade Glioma Patients Receiving TTFields Treatment: A Two Center Observational Study. Front Neurol 2019;10:1026.

Cite this article as: Liang C, Gong J, Zhang B, Meng Z, Li M, Guo Y. Multiple subtentorial metastasis in diffuse midline glioma receiving tumor treating fields: a case report and literature review. Ann Transl Med 2021;9(20):1604. doi: 10.21037/atm-21-4395
21. Shi W, Blumenthal DT, Oberheim Bush NA, et al. Global post-marketing safety surveillance of Tumor Treating Fields (TTFields) in patients with high-grade glioma in clinical practice. J Neurooncol 2020;148:489-500. Erratum in J Neurooncol 2021;151:339. 